Article

\title{
Analysis on Evolution Characteristics and Dynamic Mechanism of Urban Green Innovation Network: A Case Study of Yangtze River Economic Belt
}

\author{
Renjie Zhang ${ }^{1}$, Hsingwei Tai ${ }^{2, *}{ }^{\mathbb{D}}$, Kuo-Tai Cheng ${ }^{3}$, Zhengxu Cao ${ }^{4}$, Huizhong Dong ${ }^{5}$ and Junjie Hou ${ }^{1}$ \\ 1 China Aerospace Academy of Systems Science and Engineering, Beijing 100830, China; \\ 17865919628@139.com (R.Z.); houjj@spacechina.com (J.H.) \\ 2 School of Civil and Architectural Engineering, Shandong University of Technology, Zibo 255000, China \\ 3 Department of Environmental and Cultural Resources, National Tsing Hua University, \\ Hsinchu 300044, Taiwan; Tigercheng@mail.nd.nthu.edu.tw \\ 4 Management College, Ocean University of China, Qingdao 266100, China; caozhengxu1@163.com \\ 5 School of Management, Shandong University of Technology, Zibo 255000, China; sdutdhz@126.com \\ * Correspondence: rt007204@gmail.com; Tel.: +86-15269366192
}

Citation: Zhang, R.; Tai, H.; Cheng, K.-T.; Cao, Z.; Dong, H.; Hou, J. Analysis on Evolution Characteristics and Dynamic Mechanism of Urban Green Innovation Network: A Case Study of Yangtze River Economic Belt. Sustainability 2022, 14, 297 https://doi.org/10.3390/su14010297

Academic Editor: Brian Deal

Received: 13 November 2021

Accepted: 24 December 2021

Published: 28 December 2021

Publisher's Note: MDPI stays neutral with regard to jurisdictional claims in published maps and institutional affiliations.

Copyright: (C) 2021 by the authors. Licensee MDPI, Basel, Switzerland. This article is an open access article distributed under the terms and conditions of the Creative Commons Attribution (CC BY) license (https:// creativecommons.org/licenses/by/ $4.0 /)$.

\begin{abstract}
This study puts forward a logical framework for green innovation network analysis, which includes a spatial dimension, a relational dimension, and a systems dimension. Here, we put forward some basic research ideas concerning the optimization and regulation of green innovation networks in terms of the systems dimension and we investigate the micro-dynamic mechanisms of green innovation network expansion using a spatial econometric model. Our main research results are as follows: The efficiency of green innovation in the Yangtze River Economic Belt has improved significantly, however, the gap between cities has gradually increased, and a problem of efficiency regression has emerged. The green innovation network has changed from the primary stage dominated by Edge Network to the rapid growth stage dominated by Supporting Network, and formed a complex network pattern with diversified hierarchical structure. Node symmetry is helpful in forming more extroverted connections and promoting the expansion of green innovation networks. Node proximity and connection symmetry inhibit the growth and development of networks, and knowledge flow cooperation networks can accelerate the evolution of green innovation networks. Finally, this paper holds that we should combine the actual development needs, emphasize the basic principles of differentiated development, and construct the development pattern of regional collaborative innovation. This can also provide a theoretical reference for enriching our understanding of green innovation networks while narrowing the gap between cities.
\end{abstract}

Keywords: Yangtze River Economic Belt; green innovation network; social network analysis; gravitational model

\section{Introduction}

The Yangtze River Economic Belt is an important part of the " $\mathrm{T}$ "-shaped spatial structure of China's land development and strategic planning, and it is also an important channel connecting China with other countries around the world. Its superior geographical location and policy support make it one of the most active areas for innovation and development in China [1-3]. In 2019, investment in science and technological research and development in the Yangtze River Economic Belt reached 8466.30 billion yuan, accounting for 34\% of total investment. The total number of patent applications and authorizations reached 158,344-a nearly 5.6-fold increase compared with 2010 [4]. These data show that the Yangtze River Economic Belt has strong development potential and innovative ability. With the improvement of innovation capacity, there is also a serious environmental pollution problem, especially in terms of water pollution and carbon emissions $[5,6]$. The Yangtze River Economic Belt is an economic development area planned by the 11 provinces that run 
through the Yangtze River. The Yangtze River provides convenient shipping conditions for economic activity and trade between provinces and cities, and it attracts a large number of industrial enterprises on both sides. Chemical and traditional manufacturing industries with high pollution and high energy consumption have brought major challenges in terms of air and water quality for the Yangtze River basin [7]. In 2019, the industrial wastewater discharge in the Yangtze River Economic Belt reached 314.02 million tons, and total carbon emissions reached 3529.81 million tons.

The "black economic model", which is characterized by high levels of energy consumption and high environmental costs, is no longer suitable for the development of the Yangtze River Economic Belt. Instead, green innovation provide a new idea for compensating the environmental costs of economic growth. Green innovation describes an innovative form with environmentally friendly characteristics [8,9], and emphasizes the development and application of energy-saving and environmental protection technologies. The efficiency of green innovation quantitatively shows the rationalization degree of innovative resource allocation in a particular region from the perspective of input and output. It has laid a foundation for investigating green innovation activities in the Yangtze River Economic Belt from the perspective of space-time evolution [10]. However, the expansion of regional trade, high-speed rail, aviation, and other modes of transportation has greatly reduced the space distance between cities, resulting in the progressive strengthening of green innovation links. Using traditional "central place theory", it is difficult to effectively explain the green innovation relationships between cities-in other words, green innovation network is rooted in many fields such as economy, transportation, information, and markets among cities, and weakens the limitation of geographical space.

A green innovation network is typically a large system with the basic characteristics of openness, complexity, and nonlinearity [11,12]. The investigation of such structures in terms of micro-organizational modes and influencing factors can help to accurately characterize the green innovation links among cities in the Yangtze River Economic Belt, and also help to provide effective development plans for areas with weaker green innovation capabilities. However, the analysis of green innovation networks still lacks a comprehensive and effective logical framework. The structure of green innovation networks, as well as their behavior patterns and the micro-dynamic mechanisms of networks, have not yet been resolved.

This study will specifically explain the formation and evolution mechanism of green innovation network, and establish an analytical framework for green innovation network in view of the shortcomings of existing research, and at the same time try to decompose the micro-structure of green innovation network and discuss its micro-dynamic mechanism. We addresses the above problems in the following ways: Related forms of data, such as the number of patents granted and the comprehensive index of environmental pollution, were obtained from the databases of China Urban Statistical Yearbook (2010-2019) and the China Environmental Statistical Yearbook (2010-2019) in order to calculate the green innovation efficiency for various cities. The green innovation network is constructed using a gravity model and the indexes, such as network density and degree centrality, were investigated using this approach. Finally, the micro-dynamic mechanisms of green innovation networks were analyzed using spatial econometric modeling. Our main findings are that the efficiency of green innovation in the Yangtze River Economic Belt has been significantly improved and the intensity of green innovation networks continues to increase. The imbalance between cities and an imbalance in terms of network structure will continue to exist, and shows the group evolution trend of tripartite alliance.

\section{Literature Review}

There are various types of cities in the Yangtze River Economic Belt and the evolution of its green innovation networks is exemplary. In this sense, it can provide a model for the development of green innovation efforts in other regions. Therefore, this study begins by analyzing the relevant literature concerning green innovation and green innovation efficiency. 


\subsection{Green Innovation $\mathcal{E}$ Green Innovation Efficiency}

Green innovation is the expansion and supplementation of the concept of urban innovation under the influence of a more comprehensive consideration of economic, social, and ecological factors. This has a richer theoretical context and a deeper practical significance. Green innovation is also referred to as ecological innovation, environmental innovation, and sustainable innovation [13]. Its essence is to pay more attention to the negative impact on the sustainable development of ecological environment while considering the economic and social benefits in the process of innovation. It is the integration of various values, such as economic value, social value, humanistic value, and technical value [14,15], emphasizes the development and application of green technology, and reduces the negative externalities of environment in the process of innovation. It is also a key step in the greening and ecologicalization of urban innovation [16]. From another perspective, green innovation is also an intangible resource with which to achieve sustainable development [17-19], and thus to extend the concept of green innovation efficiency [20]. Green innovation efficiency not only reflects innovation performance under certain environmental constraints but also reflects ecological sustainability in the process of innovation and shows typical "double attributes" characteristics, which makes it an important indicator in evaluating the coordination between regional innovation and ecological economies [21]. It can be seen that there are some differences in the understanding of the concept and connotation of green innovation, but they are all based on economic development and ecological sustainability. This paper holds that green innovation is an innovation activity in the process of realizing the ecology and sustainability of economic growth, and the efficiency of green innovation reflects the effectiveness of resource allocation. The measurement of green innovation efficiency [22-24], temporal and spatial evolution [25-27], and influencing factors [28] are the main research directions of existing research. However, with the development of an urban agglomeration economic model and the implementation of a regionally coordinated development strategy, cooperation barriers generated by administrative boundaries have been gradually blurred, and the green innovation links between cities are becoming increasingly proximate, the original top-down nested urban system has been broken and the complementary advantages and resources, infrastructure sharing, intensive knowledge, and high-quality talents gathered became the main driving forces to promote the green innovation links between cities. The central place theory based on freight cost and geographical distance is difficult to explain the law of the formation of inter city green innovation network. Urban green innovation shows the trend of single center to network based on "hierarchical scale", which triggers an upsurge of research on green innovation network $[29,30]$.

\subsection{Green Innovation Networks}

The theory of flow space provides a methodological basis for the construction of urban green innovation networks. The accurate description of network relationships serves as a basis for investigating the structural complexity, evolutionary expansion, spatial directivity, and micro-self-organization effects for inter-city green innovation networks [31]. The existing research is usually based on relevant data of a certain kind and it often employs a gravity model or VAR model to simulate and calculate the green innovation relationships between cities. Relying on property rights relationships between enterprises, it is often difficult to remove the influence of enterprise scale, business type, market position, and other factors on the network relationships. This can be done, however, by reconstructing the innovative network relationships between headquarters and branches [32]. Innovation networks based on science and technology parks, enterprise clusters, and industries are usually small in scale and seriously affected by network embeddedness [21,33-35]. The theory of knowledge absorption capacity provides a theoretical foundation for the construction of innovation network between cities with the flow of high-quality talents [36]. Human capital, as a key factor to promote the expansion of innovation networks and the realization of the complexity of network structure, has a high dependence on transporta- 
tion networks while the extension of high-speed rail networks will lead to the "reverse urbanization" of innovation elements [37], which can hinder the expansion and formation of innovation networks. In addition, the links formed by navigation, air transportation, and innovation funds are also used to build innovation networks between cities and these all reflect the structural characteristics and evolutionary laws of innovation networks to some extent $[38,39]$.

\subsection{A Logical Framework for Green Innovation Network Analysis}

To sum up, existing studies mainly focus on relationship building and the evolution of network morphology and structure [40]. However, the complexity of urban green innovation networks means that it is difficult to fully capture the growth laws and internal organization structure of the whole network in a single dimension. In this respect, a more complete logical framework is still needed. In order to compensate for this deficiency, we explore a logical framework for green innovation network research, which includes geospatial, network relationships, and system dimension.

Geospatial comprises a basic dimension of urban green innovation network research and the spatial characteristics of green innovation network can be examined from the perspective of "field space" theory [41]. The research of this dimension mainly focuses on spatial distribution patterns, agglomeration characteristics, and the rationalization of the allocation of the innovation elements of regional networks at the scale of provinces, cities, and urban agglomerations (bay area, metropolitan area, etc.). However, the research of geospatial dimension still does not get rid of the hierarchical nesting analysis model of central place theory, but it reflects the influence of geographical distance on the evolution of urban green innovation spaces to a certain extent, which provides a basis for the formation of innovation networks and the analysis of relational dimensions [42]. On the basis of spatial dimension, the process of further considering the green innovation connections between cities and transforming it into a complete network topology is called relational dimension. The relational dimension complements the research content of the spatial dimension with the concept of a "green innovation flow", and endows research units with the characteristics of "double attributes", that is, cities are not only in the network relationship formed by the green innovation flow, but also cannot be separated from the influence of geographical space. Research on the relational dimension tends to focus on macro-scale analysis and micro-dynamic mechanisms in the exploration of green innovation network [36]. The former focuses on the analysis of green innovation network structure and the discussion of network form expansion. Its main task is to objectively describe the hierarchy, balance, centrality, and directionality of the whole or local network. It also summarizes and explains the evolutionary laws of green innovation network in geographical space. The latter mainly emphasizes the key factors and driving mechanisms that lead to the evolution of the morphological structure for green innovation networks (centrality, directionality, center-edge, etc.). The essential attributes and embeddedness of green innovation networks determine that the influence of node attributes, network structure, and external collaborative network must be considered [43].

Spatial and relational dimensions, as well as the novel phenomena emerging from their interaction and mutual influence, constitute the system dimensions that emphasize the analysis of regulation and the optimization of green innovation networks from the perspective of system science. At present, there is little research on green innovation networks from a systems perspective. We especially point out the new characteristics of green innovation network under the system dimension. For example, besides the flow of personnel, capital, and other elements, the innovative links among innovative enterprises will also form a credit system and contract spirit in the long-term cooperation process, and the policy documents and institutional systems formulated by local governments to promote the development of innovative industrial clusters. These new characteristics have gone far beyond the essential needs of innovation cooperation among subjects, but they play an important role in ensuring services, improving quality, and increasing efficiency. 
The core task is the analysis, optimization, and regulation of regional green innovation systems and this paper provides some general steps to explore green innovation networks from the perspective of the system dimension (Figure 1).

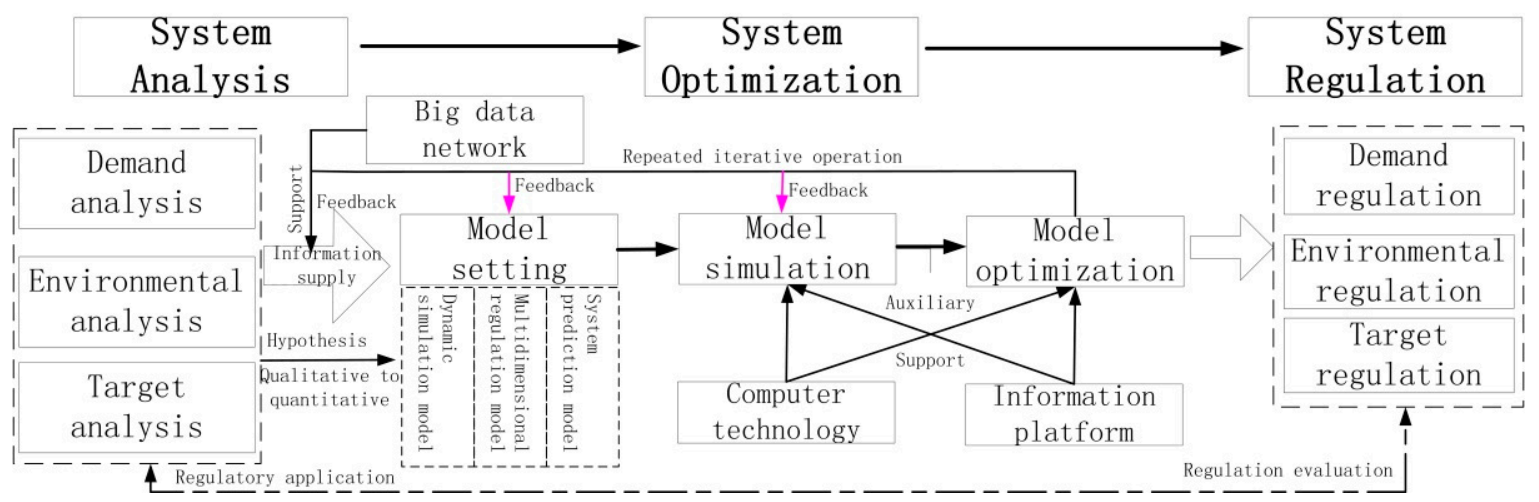

Figure 1. Simulation and controls ideas of green innovation system.

System dimension analysis can be a basis for green innovation network analysis, including system demand analysis, environment analysis and target analysis. Demand analysis focuses on the bottleneck problem in the development of green innovation system and provides an in-depth investigation of the problems faced by the innovation subject, including imperfect systems, low market flexibility, and a lack of supervision mechanisms. Environmental analysis includes attention to internal and external environments. The external environment can be further subdivided into its economic, social, ecological, environment, and market contexts. The internal environment mainly refers to a system composed of various subsystems and novel phenomena emerging between those subsystems. Environmental analysis is the key to determining system impact factors and building system simulations for the purposes of optimization. Goal analysis refers to the effect that the green innovation network will achieve through system simulation and optimization. By combining qualitative analysis with quantitative analysis, a model containing the characteristics of green innovation network is built by means of big data and digital twinning, and the optimal scheme of the system is explored through simulation optimization. System regulation and control is a specific improvement measure to optimize the green innovation system according to the simulation results, and objectively evaluates the implementation effect of the scheme and provides reference for decision-making. In addition, we summarized three empirical models for the regulation and optimization of green innovation system. The purpose of the system prediction model is to reflect the development trend of green innovation in a certain region, and it is an effective method to explain the system evolution from a macro perspective. Multidimensional regulation model and dynamic simulation model reveal the internal operation mechanism of green innovation system and provide decision support for system optimization, which are the key contents of green innovation system research.

\section{Methods and Data}

\subsection{Research Method}

\subsubsection{The Undesirable Outputs SBM Model}

(1) Model expression

Undesirable outputs SBM model is a universal tool for efficiency measurement [22-24]. Its advantage is that it considers the unexpected outputs resulting from green innovation, it avoids the influence of radial and angle on the accuracy of measurement results in 
traditional models, and it has greater applicability and scientific rigor in terms of efficiency measurement. The specific formula is as follows:

$$
\begin{aligned}
& \rho^{*}=\frac{1-\frac{1}{K} \sum_{k=1}^{K} \frac{s_{k}}{x_{k 0}}}{1+\frac{1}{I+J}\left(\sum_{i=1}^{I} \frac{s_{i}^{d}}{y_{i 0}^{d}}+\sum_{i=1}^{J} \frac{s_{i}^{u}}{y_{i 0}^{u}}\right)} \\
& \text { s.t }\left\{\begin{array}{l}
x_{k 0}=\sum_{m=1}^{M} \mu_{m} x_{k m}+s_{k}^{-}, k=1,2, \cdots, K \\
y_{i 0}^{d}=\sum_{m=1}^{M} \mu_{m} y_{i m}^{d}-s_{i}^{d}, i=1,2, \cdots, I \\
y_{i 0}^{u}=\sum_{m=1}^{M} \mu_{m} y_{i m}^{u}+s_{i}^{u}, i=1,2, \cdots, J \\
1=\sum_{m=1}^{M} \mu_{m} \\
\mu_{m} \geq 0, s_{k}^{-} \geq 0, s_{i}^{d} \geq 0, s_{i}^{u} \geq 0
\end{array}\right.
\end{aligned}
$$

where $\rho *$ represents the efficiency of urban green innovation with a value range of $[0,1] ; K$, $I$ and $J$ represent the number of green innovation inputs, the expected outputs, and the unexpected outputs respectively; $s_{k}^{-}, s_{i}^{d}$ and $s_{i}^{u}$ are the relaxation variables of innovation inputs, the expected outputs, and unexpected outputs respectively; $s_{k 0}, y_{i 0}^{d}$ and $y_{i 0}^{u}$ are the innovation inputs, expected outputs, and unexpected outputs respectively; and $\mu_{m}$ is the weight coefficient.

(2) Index selection

In this study, green innovation efficiency was chosen as the benchmark data for establishing green innovation links between cities. The reason for this is that green innovation efficiency does not only reflect the rationalization of the allocation of green innovation resources in regional cities, but it also describes the ability to transform innovation achievements into market products or services [21]. In other words, green innovation can also be regarded as the sum of all things that can help improve the urban ecological environment and achieve sustainable development, for example, the proposal of a new ecologicaleconomic governance theory or the development of new green technologies. Considering the above problems, it is particularly important to construct a logical measurement index system. Referring to the existing research, combined with the actual situation of green innovation in the Yangtze River Economic Belt $[4,19,24]$, and considering the diversified characteristics of input and output in the process of green innovation, the index system shown in Table 1 is established. The index system covers the main links of green innovation activities, in which the input includes human capital $\left(I_{1}\right)$, natural resources $\left(I_{5}\right)$ and capital $\left(I_{2}, I_{3}, I_{4}\right)$, the expected output index includes economic benefits $\left(E_{1}, E_{2}\right)$ and product benefits $\left(E_{3}\right)$, and the unexpected output consists of product risks $\left(U_{1}\right)$ and negative externalities of environment $\left(U_{2}\right)$. Especially, it shows that the accounting scope of comprehensive index of environmental pollution includes wastewater, waste gas, and solid waste, which is calculated by entropy weight method $[14,15]$. Among them, the accounting scope of wastewater refers to the total amount of sewage generated in the process of green innovation, the accounting scope of waste gas includes the total amount of sulfur dioxide, nitrogen oxides and dust particles, and the accounting scope of solid wastes mainly includes general solid wastes, such as solid particles and waste products. 
Table 1. Index system for measuring green innovation efficiency.

\begin{tabular}{ccc}
\hline Target Layer & Variable Code & Variable Meaning \\
\hline Input & $I_{1}$ & number of R\&D personnel \\
& $I_{2}$ & investment of R\&D funds \\
& $I_{3}$ & government investment in finance \\
& $I_{4}$ & number of new product development projects \\
& $I_{5}$ & total energy consumption \\
\hline Expected output & $E_{1}$ & patent authorization amount \\
& $E_{2}$ & turnover in technology market \\
& $E_{3}$ & number of papers published \\
\hline \multirow{2}{*}{ Unexpected output } & $U_{1}$ & patent unauthorized amount \\
& $U_{2}$ & comprehensive index of environmental pollution \\
\hline
\end{tabular}

\subsubsection{Gravity Model}

Both technology diffusion theory and the new economic geography theory point out that geospatial distance will hinder green innovation relationships between cities. However, with ongoing improvements to high-speed rail, aviation, and other fast transportation networks, the constraint of geospatial space on green innovation networks is obviously reduced [18]. Still, there is a significant correlation effect between the economic potential difference between cities and the expansion of green innovation networks, which requires that the influence of economic distance and geographical distance be considered simultaneously in the process of establishing green innovation relationships between cities [34]. Therefore, this paper modifies the original form of the gravity model as follows:

$$
Q_{i j}=\frac{F_{i} \times F_{j}}{D_{i j}^{2} E_{i j}^{2}} R_{i} K
$$

where $Q_{i j}$ indicates the intensity of green innovation association between city $i$ and city $j ; F_{i}$ and $F_{j}$ represent the green innovation efficiency of city $i$ and city $j$ respectively $(F$ equivalent to $\rho *$ in Formula 1$) ; D_{i j}$ and $E_{i j}$ respectively represent the geographical distance and economic distance between city $i$ and city $j$. The geographical distances in the gravity model were calculated as the linear distances after vectorization of the map. The economic distance is calculated according to the difference of GDP between the two cities [2]; $R_{i}$ represents the full-time equivalent of R\&D personnel in the city $i$; $K$ is the correction coefficient, which is used to adjust the data magnitude and enhance contrasts [2], its value selection has little effect on the subsequent result analysis.

\subsubsection{Characteristics of Green Innovation Network}

According to the different research purposes, there are also differences in the selected network characteristic indicators. In this study, network density and access degree are selected as the main indicators, aiming at revealing the structural complexity and connection direction of green innovation networks. Network density refers to the ratio between the actual number of connections and the theoretical maximum number of connections in the green innovation network. The larger the value, the stronger the relevance of the green innovation network. The specific formula is as follows:

$$
C_{R K}=\frac{S^{\prime}}{S}
$$

where: $C_{R K}$ represents the network density of the green innovation network. $S^{\prime}$ and $S$ respectively represent the actual number of connections and the theoretical maximum number of connections. 
Out-degree and in-degree respectively indicate the proportion of the number of connections with a specific node as the starting point and the end point to the total number of connections. The specific formula is as follows:

$$
\begin{aligned}
C_{i, \text { in }} & =\frac{L_{\text {in }}}{\left(L_{\text {in }}+L_{\text {out }}\right)} \\
C_{i, \text { out }} & =\frac{L_{\text {out }}}{\left(L_{\text {in }}+L_{\text {out }}\right)}
\end{aligned}
$$

where $C_{i, \text { out }}$ and $C_{i, \text { in }}$ respectively represent the out-degree and in-degree values of node $i$, and $L_{\text {out }}$ and $L_{i n}$ respectively represent the number of connections with node $i$ as the starting and the end.

\subsubsection{Spatial Econometric Model}

\section{(1) Spatial Lag Model}

The Exponential Random Graph Model (ERGM) and QAP regression are common tools for investigating the factors influencing network, though they can only deal with cross-sectional data. Moreover, it is difficult to build such models and the results are often unsatisfactory in terms of the iterative operational process. In this study, a spatial econometric model is used to investigate the micro-dynamic mechanisms of green innovation networks during the whole cycle. The specific model is as follows:

$$
\ln C_{i, t}=\beta_{0}+\rho W \ln C_{i, t-1}+\beta_{2} X_{i}+\alpha_{i}+\gamma_{i}+\varepsilon_{i, t}
$$

where $C_{i, t}$ represents the comprehensive index of the green innovation network. From Formula 3, we can see that green innovation efficiency, innovation investment, and economic distance are key factors affecting green innovation networks between cities, and changes in their values directly affect the complexity and expansion of the network structure.

Therefore, this paper uses the comprehensive index to describe key factors in the evolution of green innovation network structures, and $C_{t-1}$ is the comprehensive index that lags 1 period behind; $\rho$ represents spatial autocorrelation coefficient; $W$ represents the spatial weight matrix; $X$ represents an explanatory variable; $\alpha_{i}$ and $\gamma_{i}$ represent individual fixed effect and time fixed effect; and $\varepsilon_{i, t}$ is a random interference term.

(2) Variable Selection

Previous studies have found that a city's own attributes, network microstructure, and other external networks have an impact on the expansion of green innovation networks $[14,16]$. Table 2 shows the selected explanatory variables and describes the meaning of each variable.

Note: each variable in Table 1 can be calculated by the software Ucinet. $G_{\text {out }}$ and $G_{\text {in }}$ represent the out- degree and in-degree values of the green innovation network, respectively, and $q_{i}$ represents the green innovation correlation values of all nodes associated with the node $i$.

Node symmetry $\left(X_{1}\right)$ is used to reflect the convergence and divergence of network nodes. The greater the deviation is from 1 , the worse the symmetry of the node is. Among these, a positive deviation (value greater than 1) shows divergent nodes, while a negative deviation (value less than 1 ) shows convergent nodes. Node proximity $\left(X_{2}\right)$ can reflect the relative size of the distance and the average distance between the target node and the remaining nodes. If the value is greater than 1 , it means that the path traversed by a particular node through the remaining nodes is greater than the average distance. This kind of node usually exhibits long-distance connections. Otherwise, short-distance connections are more prevalent. Connection efficiency $\left(X_{3}\right)$ can effectively describe the ratio of the connection strength between the target node and other nodes in terms of the average connection strength. The greater the connection efficiency, the more stable the connection relationship between the two nodes and the stronger the support for the network structure. Otherwise, the connection relationship is more fragile. Connection symmetry $\left(X_{4}\right)$ is used to reflect the relative strength of the receiving and sending connections of target nodes. The 
difference between connection symmetry and node symmetry is that the former emphasizes the breadth of the connections of target nodes, while connection symmetry focuses on the depth of connection with other nodes. In this paper, knowledge flow networks $\left(X_{5}\right)$ are constructed according to the cross-city flow of high-quality personnel and its influence on green innovation network is investigated by taking the sum of the connections between networks as co-network factors.

Table 2. Explanatory variables of spatial econometric model.

\begin{tabular}{|c|c|c|c|c|}
\hline Type & Variable & $\begin{array}{l}\text { Variable } \\
\text { Code }\end{array}$ & $\begin{array}{c}\text { Computational } \\
\text { Formula }\end{array}$ & Variable Interpretation \\
\hline \multirow{2}{*}{$\begin{array}{l}\text { Node } \\
\text { attribute }\end{array}$} & $\begin{array}{c}\text { Node } \\
\text { symmetry }\end{array}$ & $X_{1}$ & $X_{1}=\frac{G_{o u t}}{G_{i n}}$ & $\begin{array}{l}G_{\text {out }} \text { and } G_{\text {in }} \text { represent node outputs and inputs, } \\
\text { respectively. Larger ratios indicates that the } \\
\text { nodes diverge; otherwise, the nodes converge. }\end{array}$ \\
\hline & $\begin{array}{l}\text { Node } \\
\text { proximity }\end{array}$ & $X_{2}$ & $X_{2}=\frac{N P_{i}}{\sum_{j=1}^{N} P_{j}}$ & $\begin{array}{l}P_{i} \text { and } \bar{P} \text { represent the closeness and average } \\
\text { closeness of node } i \text {, respectively. The larger the } \\
\text { value, the farther away it is from other nodes, } \\
\text { and the closer it is to other nodes. }\end{array}$ \\
\hline \multirow[b]{2}{*}{ Network structure } & $\begin{array}{l}\text { Connection } \\
\text { efficiency }\end{array}$ & $X_{3}$ & $X_{3}=\frac{N \sum_{i=1}^{N} q_{i}}{\sum_{i=1}^{N} \sum_{i=1}^{N} q_{i}}$ & $\begin{array}{l}q_{i} \text { represents the total strength of green } \\
\text { innovation connections to node } i \text {. The larger the } \\
\text { value, the more stable the network structure; } \\
\text { otherwise, the more fragile it is. }\end{array}$ \\
\hline & $\begin{array}{l}\text { Connection } \\
\text { symmetry }\end{array}$ & $X_{4}$ & $X_{4}=\frac{S_{\text {in }}}{S_{\text {out }}}$ & $\begin{array}{l}S_{i n} \text { and } S_{\text {out }} \text { represent the sum of receiving } \\
\text { intensity and sending intensity for the nodes, } \\
\text { respectively. The smaller the value, the more } \\
\text { active the nodes are in the network, showing } \\
\text { spillover effects. Otherwise, this shows a } \\
\text { siphon effect. }\end{array}$ \\
\hline $\begin{array}{l}\text { Knowledge flow } \\
\text { network }\end{array}$ & $\begin{array}{c}\text { Collaborative } \\
\text { network connection }\end{array}$ & $X_{5}$ & $X_{5}=\sum_{i=1}^{N} M_{i}$ & $\begin{array}{l}M_{i} \text { represents the connection strength of the } \\
\text { target node to the node. }\end{array}$ \\
\hline
\end{tabular}

\subsection{Data Exploration}

In this study, 2010-2019 was selected as the study period and the relevant data were collected from the China Urban Statistical Yearbook (2010-2019), the China Environmental Statistical Yearbook (2010-2019), and the China Urban Database (http: / / olap.epsnet.com.cn, accessed on 21 July 2021). The map resources are taken from the Standard Map Service System (http:/ / bzdt.ch.mnr.gov.cn/, accessed on 9 July 2021) and the geographical distances in the gravity model were calculated as the linear distances after vectorization of the map. The economic distance is calculated according to the difference of GDP between the two cities [2].

Checking and cleaning the collected data was an important step of this process in ensuring the accuracy of our results, and a key task was to eliminate possible outliers. After inspection, the standard deviation of the green innovation efficiency measurement index data was within acceptable limits, and 15,480 green innovation efficiency values for 129 cities were obtained. On this basis, a gravity model was used to calculate the correlation strength between cities, thus forming a $129 \times 129$ green innovation correlation matrix. Our results show that there are many values in this matrix far below the mean. In order to avoid undue influence from this kind of data, the furthest outlying values were eliminated and the green innovation correlation matrix of $110 \times 110$ was obtained after this correction.

\section{Results}

\subsection{Analysis of Green Innovation Efficiency Results}

The green innovation efficiency of Yangtze River Economic Belt from 2010 to 2019 was calculated using an SBM model. Table 3 shows the descriptive statistics for green 
innovation efficiency, which were used to compare trends and the degree of differentiation for overall green innovation efficiency in the Yangtze River Economic Belt at different times. Using ArcGIS software to visualize the green innovation efficiency, Figure 2 shows the spatial distribution of green innovation efficiency values.

Table 3. Descriptive statistics for green innovation efficiency in Yangtze River Economic Belt.

\begin{tabular}{cccccccccc}
\hline Year & Max & Min & Ave & Ran & C.V & Var & Std & Ske & Kur \\
\hline 2010 & 0.864 & 0.152 & 0.323 & 0.712 & 1.578 & 0.262 & 0.511 & 0.430 & 0.228 \\
2013 & 0.890 & 0.167 & 0.320 & 0.723 & 1.691 & 0.293 & 0.541 & 0.456 & 0.259 \\
2016 & 0.917 & 0.182 & 0.347 & 0.742 & 1.713 & 0.353 & 0.594 & 0.525 & 0.346 \\
2019 & 0.990 & 0.219 & 0.385 & 0.771 & 1.732 & 0.445 & 0.667 & 0.636 & 0.495 \\
\hline
\end{tabular}
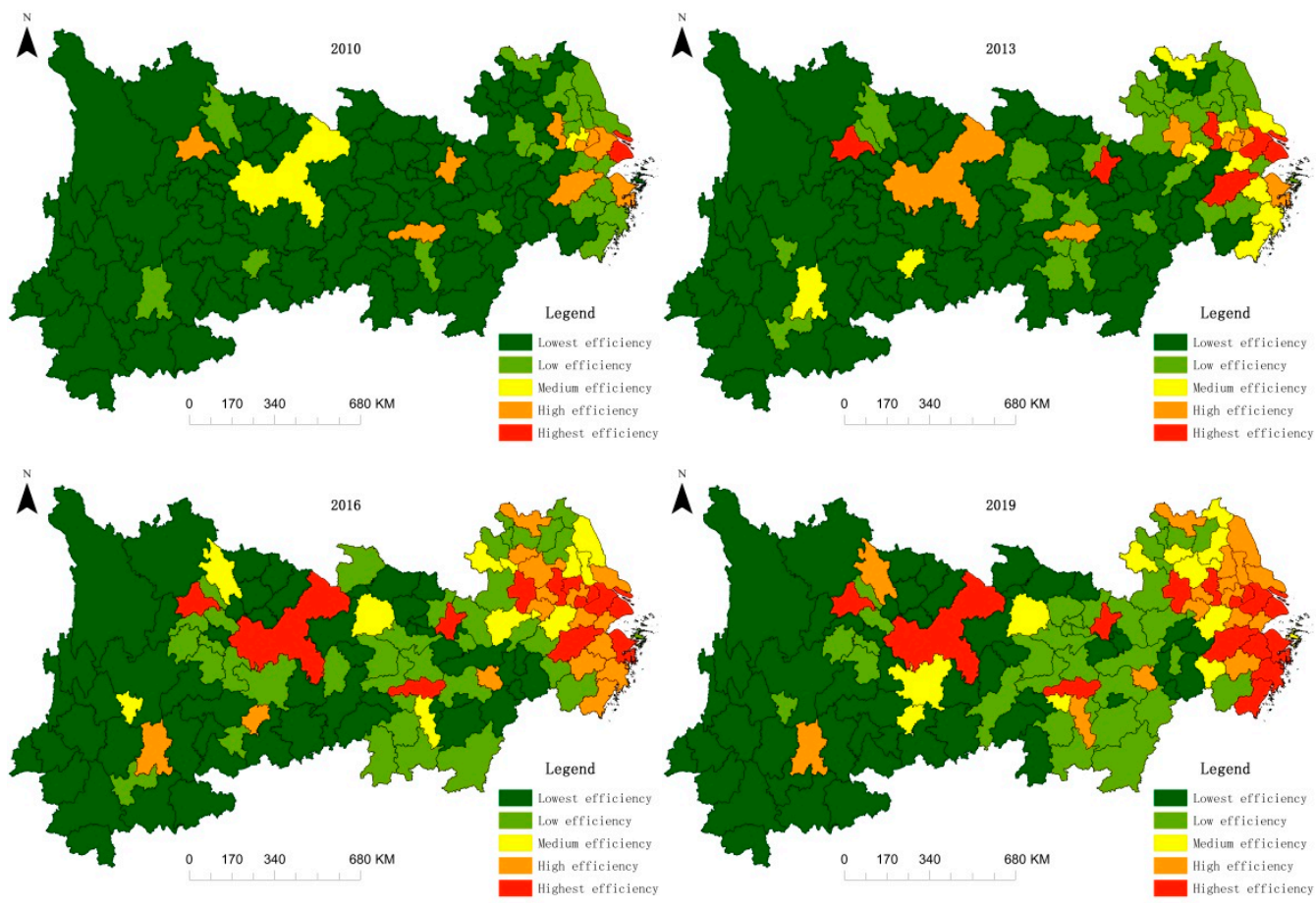

Figure 2. Spatial distribution pattern of green innovation efficiency in Yangtze River Economic Belt.

\subsubsection{Descriptive Statistics for Green Innovation Efficiency}

The descriptive statistics for green innovation efficiency are shown in Table 3. Coefficient of variation (C.V) values are useful for comparing the degree of dispersion for green innovation efficiency during different years. Skewness (Ske) is used to characterize the asymmetry of the probability distribution for green innovation efficiency values and kurtosis (Kur) is used to characterize the steepness of green innovation efficiency distribution.

It evident from Table 3 that the average (Ave) efficiency of green innovation in the Yangtze River Economic Belt is slowly increasing, with an average annual growth rate of around $6 \%$, with both maximum (Max) and minimum (Min) efficiency values increasing slightly. The coefficient of variation (C.V) also shows a continuous upward trend. With the passage of time, the green innovation efficiency for cities in the Yangtze River Economic Belt is split, and the heterogeneity of inter-city innovation resource allocation and platform service construction appears to have led to a widening gap in terms of green innovation efficiency. From the changes of standard deviation (Std) and range (Ran), we can conclude that the overall difference in green innovation efficiency in the Yangtze River Economic Belt is growing over time. Combined with the changes in variance (Var), it is evident that the distribution for green innovation efficiency is divided among two poles around the mean value, though it is not significantly polarized in a formal sense. The 
skewness (Ske) and kurtosis (Kur) values show that the overall efficiency distribution forms a left-leaning, long-tailed sample structure, and the high proportion of inefficient cities has become a bottleneck problem, which hinders the overall improvement of green innovation efficiency in the Yangtze River Economic Belt and which narrows the gap of green innovation among regions.

\subsubsection{Green Innovation Efficiency Shows a Dynamic Spatial Distribution Pattern}

The efficiency of green innovation in the Yangtze River Economic Belt improved significantly over the course of research period. The number of cities classified as either High Efficiency or Highest Efficiency has increased from 9 in 2010 to 30 in 2019. Specifically, only Shanghai reached the Highest Efficiency level in 2010, showing a spatial distribution pattern in which one pole is dominant. The provincial capitals, such as Suzhou, Hangzhou, Chengdu, and Changsha, all belong to the High Efficiency category. In 2013, these cities also started to transition to the Highest Efficiency level and the number of cities in the Lowest Efficiency category decreased significantly. At that time, cities with Medium and High Efficiency levels began to appear in the middle and upper reaches of the Yangtze River Economic Belt, which provided strong support for driving weaker cities in terms of green innovation and elevating efficiency values at the lower end of the distribution. Until 2016, the spatial pattern of green innovation efficiency in the middle and upper reaches of the Yangtze River Economic Belt showed strong dynamics. It is worth noting that the dynamic spatial pattern of urban green innovation efficiency in the Yangtze River Economic Belt decreased rapidly in 2019, with only a few Low Efficiency-level cities growing and even some instances of efficiency degradation (Chuzhou, Bengbu, Panzhihua, etc.). This change means that the green innovation efficiency of cities in the Yangtze River Economic Belt appears to have reached a growth bottleneck at that time.

The location endowment and strategic position of the Yangtze River Delta region results in the fact that it has more abundant innovation resources. However, with the improvement of the domestic innovation environment and changes in market demand, the green innovation growth driven by factor inputs gradually loses its primacy. The redundancy of innovation elements, inefficiency of organization and management, and lack of supervision and supervision system have become elements of a "ceiling" hindering the improvement of green innovation efficiency; Cities in the middle and upper reaches of the Yangtze River Economic Belt still tend to be at the Low Efficiency level for green innovation, and there are still constraints in terms of innovation elements and economic scale. Therefore, improving the efficiency of green innovation in the Yangtze River Economic Belt must be carried out according to the idea of adapting to local conditions, regional linkages, and dislocations in the division of labor. That is, based on the bottleneck problem faced by green innovation in different regions, establishing and perfecting regional linkage mechanisms is crucial in ensuring the free and smooth flow of elements and information in order to create a green innovation pattern with dislocation in the division of labor.

\subsubsection{The Efficiency of Green Innovation Shows a Stubborn and Heterogeneous Evolution}

The efficiency of green innovation has always shown a spatial imbalance across the entire research period, forming a step-like morphological feature that the upstream region is a high-value region and gradually decreases to the upstream region. The global Moran'I increased from 0.027 in 2010 to 0.367 in 2019, and both were significant at the $10 \%$ confidence level. This indicates that the spatial evolution pattern for green innovation efficiency is not randomly distributed, and the degree of interdependence and mutual influence between cities is gradually strengthening. However, the laws of local agglomeration are characterized by and unbalanced feature in which the lowest values surround the highest values. In other words, there is an agglomeration phenomenon with the provincial capital or central city as the core and this fosters innovation spillover effects for the city with the highest level of efficiency. 
4.1.4. Spatial Change in Green Innovation Efficiency in Local Areas Shows Heterogeneity and Graduality

The Yangtze River Delta region has changed from a Shanghai-centered single-core pattern to a Shanghai-Suzhou-Hangzhou multi-core spatial layout. In this way, it has formed a concentrated and contiguous green innovation high-efficiency area, which has experienced three stages of efficiency: a lower-efficiency balance, a medium-efficiency imbalance, and a high-efficiency imbalance. The middle reaches of the Yangtze River have always shown the characteristics of dependence on the provincial capital city, which resulted in a trend of lowest efficiency equilibrium, lower efficiency imbalance, lower efficiency equilibrium in its process of evolution. Similarly, the Chengdu-Chongqing region also shows a similar evolution. By comparison, it is found that the upstream region has not played a leading role in the radiation of innovation in Chengdu and Chongqing, which has led to a slow spatial evolution in terms of green innovation efficiency.

\subsection{Evolutionary Analysis of Green Innovation Network Structure}

We can now visualize the corrected green innovation association matrix. Figure 3 shows the spatial structure of the green innovation association network. In order to investigate growth-and-development rules and the structural evolution characteristics of the green innovation network, this can be divided into four levels of sub-networks according to the correlation strength between cities as follows: Core Network; Support Network; Basic Network; and Edge Network. The Core Network refers to the sub-network composed of cities with green innovation correlation intensity exceeding 3164.26, which has a strong spillover effect on driving the green innovation development of a certain region (metropolitan area and urban agglomeration). This is also the key to promote the complexity and advancement of green innovation-related networks. The correlation intensity of urban green innovation in the Support Network is in the range of [1934.34, 3164.26], which reflects the transition state of the green innovation network to the core network and also determines the evolution direction of the whole network. The connection intensity of urban green innovation in the Basic Network is in the range of [524.34, 1934.34], which represents the general and universal connection in the green innovation network. The Edge Network refers to the sub-network composed of cities with less than 524.34 green innovation correlation intensity, which reflects the weak ties in the network and the development potential of the network from the side.
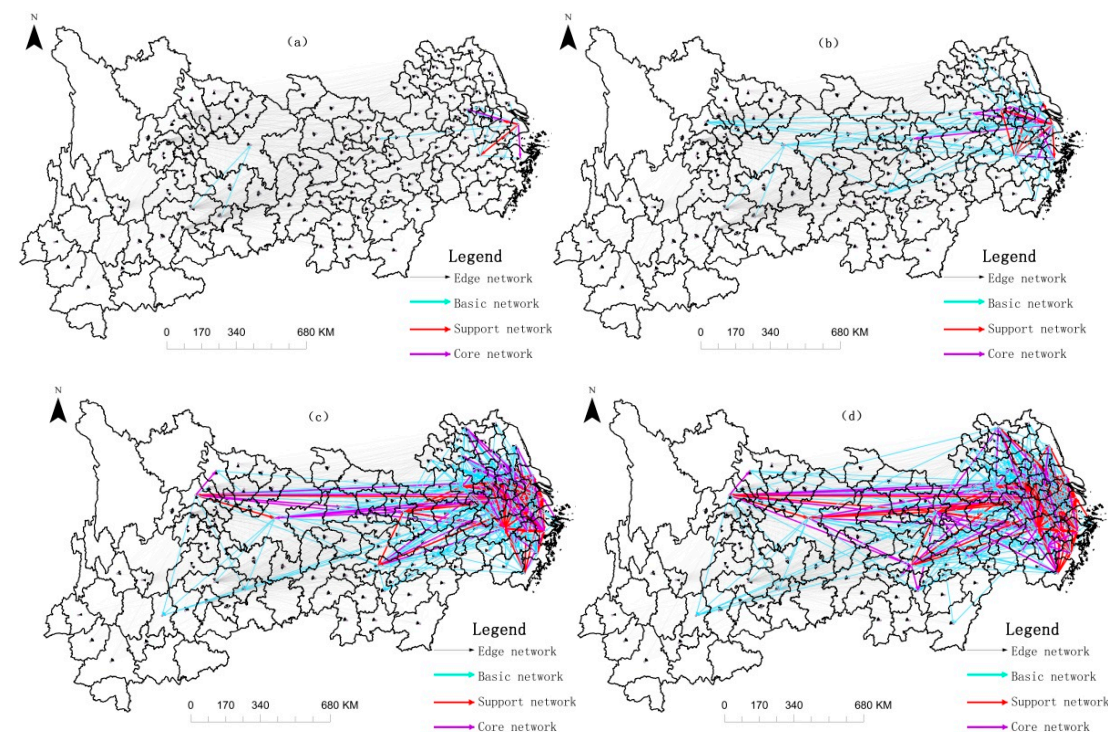

Figure 3. Structural characteristics of the urban green innovation network in Yangtze River Economic Belt. (a) Network structure of green innovation in 2010; (b) Network structure of green innovation in 2013; (c) Network structure of green innovation in 2016; (d) Network structure of green innovation in 2019. 


\subsubsection{The Density of the Green Innovation Network Has Increased Significantly}

In 2010, the network density was only 0.013 , and the green innovation network was at the initial stage of growth and development dominated by Edge Network characteristics of simplicity and sparsity. With the passage of time, a Basic Network structure began to predominate, and the green innovation network began to enter a period of rapid growth. Before 2019, network density rose to 0.164 , initially forming a green innovation network pattern with diversified structures. However, there are still a large number of isolated nodes in terms of network density, which provides room for development in terms of the extension of the green innovation network.

\subsubsection{The Green Innovation Network Has an Unbalanced Structure and Level}

In 2010, a strong connection across the green innovation network only appeared among Shanghai, Hangzhou, Nanjing, and other major cities. Beginning in 2013, the complexity of the green innovation network in the Yangtze River Delta region was further enhanced, and the basic network connection began to appear in the river's middle and upper reaches. It is worth noting that Chengdu-Chongqing area has not been connected with nearby areas, but rather to points in the Yangtze River Delta region that are farther away. Therefore, it can be inferred that the green innovation network of cities in the Yangtze River Economic Belt has the characteristics of preference attachment, and it is easier for cities with similar attributes to form network connections. It also shows that the expansion of the green innovation network gradually breaks the barriers of geographical distance and administrative boundaries, and this change will be more conducive to the integration of new urban nodes into the green innovation network, as well as promoting the complexity and advancement of network structure. With the passage of time, the green innovation network has further expanded to the middle and upper reaches of the region, forming a horizontal " $\mathrm{T}$ "-shaped spatial structure. Sone spatial imbalance always exists, especially in Guizhou, Yunnan, and western Sichuan, which is mainly due to tourism and agricultural development in this area. It is difficult to effectively embed within the overall green innovation network due to constraints of industrial support and insufficient endogenous motivations for green innovation. The imbalance in terms of hierarchical structures is mainly reflected in the low total connection strength with the Edge Network category, though it accounts for a large proportion of the total number of connections. Therefore, reducing the proportion of Edge Networks cases among the overall network, eliminating redundant connections between cities, and developing support networks and core networks are effective ways to achieve the balanced development of a green innovation network hierarchy.

\subsubsection{Micro-Self-Organization Structures Promote the Expansion of the Green Innovation Network}

Combined with the analysis of the micro-configuration calculation results for green innovation efficiency in Table 4, Type (1) indicates that there are no isolated nodes connected by green innovation and the number of instances belonging to this structure has dropped significantly. This reflects the fact that more urban nodes are included in the green innovation network, which is intuitively manifested by the improvement of network density. Type (2) represents the basic connection form for the green innovation network between cities and it is also the basis for network structure expansion. Type (3) reflects the two-way connection of green innovation network relationships between cities and the reciprocal characteristics of network connections. The sudden increase in its prevalence means that the green innovation network is complicated and advanced. Types (4) and (5) are interpreted as the divergence (out-2-star) and the agglomeration (in-2-star) of the green innovation network, respectively. It is evident from their quantitative changes that the expansion of the green innovation network is far greater than that of agglomeration. Type (6) is the situation in which the in-2-star contains a special form of preference attachment, which expresses a more complex tripartite relationship. Types (7) and (8) reflect a context 
in which the green innovation network has the tendency for group relationship evolution

based mainly on closed tripartite association.

Table 4. Micro-configuration of the green innovation network.

\begin{tabular}{|c|c|c|c|c|c|c|c|}
\hline Type & Network Configuration & 2010 & 2019 & Type & Network Configuration & 2010 & 2019 \\
\hline (1) & & 19,949 & 10,564 & (5) & & 553 & 6712 \\
\hline (2) & & 13,064 & 26,549 & (6) & & 192 & 3648 \\
\hline (3) & & 1525 & 30,220 & (7) & & 152 & 4613 \\
\hline (4) & & 480 & 13,511 & 8 & 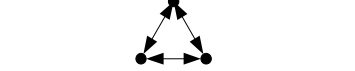 & 8 & 2120 \\
\hline
\end{tabular}

\subsection{Analysis of the Dynamic Mechanisms of the Green Innovation Network}

The regression test of the spatial lag model was carried out using the Stata14.0 software package. In order to validate the results of the model, we choose the fixed effect model for time and individual. By testing the Log-likelihood and adjusted R-square values, we find that the spatial lag model has more advantages and better robustness than linear regression in explaining the micro-dynamic mechanisms of green innovation network expansion. Table 5 shows the regression results of the model.

Table 5. Regression results.

\begin{tabular}{ccc}
\hline Variable & OLS & SLM \\
\hline$X_{1}$ & 0.061 & $0.167^{* *}$ \\
$X_{2}$ & $0.350^{*}$ & $-0.224^{* * *}$ \\
$X_{3}$ & $0.014^{*}$ & $0.064^{*}$ \\
$X_{4}$ & $0.035^{* *}$ & $-0.351^{* *}$ \\
$X_{5}$ & 0.164 & $0.235^{*}$ \\
R2-ad & 0.420 & 0.696 \\
Log-likelihood & - & 264.126 \\
Individual effect & Control & Control \\
Time effect & Control & Control \\
Time effect & 1100 & 1100
\end{tabular}

Note: ${ }^{*},{ }^{* *},{ }^{* * *}$ mean significant at $1 \%, 5 \%$ and $10 \%$ confidence levels.

According to our analysis of regression results, the regression coefficient for node symmetry is 0.167 , which is significant at $10 \%$ confidence level. This indicates that the node symmetry has a positive role in promoting the expansion of the green innovation network. The symmetry of the nodes is the result of the game between out-degree and in-degree. Divergent nodes tend to form more extroverted connections, and the expansion of the green innovation network is realized by absorbing isolated or marginal nodes. These usually have a high network status and influence, while convergent nodes focus on receiving connections from other nodes, which results in path locking and structural rigidity for the green innovation network. This is often the case in the marginal areas of the green innovation network.

Node proximity can inhibit the growth and development of the green innovation network, and a high level of node proximity leads to an increase in the distance spent on green innovation network connections, which reduces the network's transmission efficiency. The regression coefficient for connection efficiency is 0.064 , which is significant at $10 \%$ confidence level. This shows that connection efficiency can promote the expansion of the green innovation network and a high level of connection efficiency exists mainly between core nodes of the green innovation network. In this way, it is helpful in establishing a more 
stable network architecture. The regression coefficient for connection symmetry is -0.351 , which is significant at $5 \%$ confidence level. This indicates that connection symmetry is not conducive to the expansion of the green innovation network and a siphon effect is helpful in the formation of new core nodes. However, it will also lead to the loss of the innovation elements of neighboring nodes and increase their own hierarchy. The regression coefficient for knowledge flow in the network is 0.235 , which is significant at $10 \%$ confidence level. This indicated that knowledge flow can promote the growth and development of green innovation network. The cross-city flow of high-quality personnel will promote the exchange and integration of new knowledge, experience, and technology. It will also help to enhance the core competitiveness of urban green innovation, thus accelerating the expansion of the green innovation network.

\section{Policy Implications}

The government always plays a dual role of manager and participant in the green innovation development of the Yangtze River economic belt. Policy system and market mechanism are the key factors affecting regional green development. Combined with the above research results, the following targeted policy suggestions are put forward to promote green innovation in the Yangtze River Economic Belt.

It is beneficial to focus on the planning and layout of high-tech industries represented by new energy and new materials, equipment manufacturing, biomedicine and big data in the middle and upper reaches of the study area. In addition, it useful to encourage and support the flow of innovation elements to the region and to promote the formation of a green innovation pattern with a misplaced division of labor. This can fill network structure depressions, such as those in Yunnan and Guizhou, and narrow the gap in terms of green innovation between regions. With the implementation of distributed innovation in relation to local conditions, the Yangtze River Delta urban agglomeration continues to play a leading role in the field of green innovation and it actively absorbs and introduces advanced international technology. In relying on geographical advantages, it is constantly improving its capability of independent innovation while focusing on breaking through the blockade of technologies with strategic significance, such as lithography machines and nano chips.

At the same time, this region relies on the shipping advantages of the "golden waterway" of the Yangtze River and the transportation networks such as the Shanghai-Kunming Expressway and its riverside channels. This promotes the agglomeration of relevant technologies, personnel, and resources to the middle and upper reaches. The middle reaches should actively undertake the industrial transfer from the Yangtze River Delta, and work at the ongoing improvement of its level of urban green innovation. This can provide strong support for the innovation and development of the western region. Due to the constraints on leading industries, the Yunnan-Guizhou-Sichuan region has always been on the edge of the green innovation network. We should actively promote and introduce innovative industries and improve the endogenous driving forces of green innovation. For example, we should carry out the research and development on the core technologies having to do with new energy sources, such as wind and solar energy. We should encourage the development of the green tourism industry and promote the application of ecological agriculture technology. At the same time, our findings emphasize the complementary role of small-sized and medium-sized enterprises in terms of green innovation, which supports and encourages the upgrading of basic innovative technologies.

\section{Conclusions and Prospects}

This paper tentatively puts forward a logical framework for the analysis of green innovation networks from the perspectives of spatial, relational, and system dimensions, and it puts forward some basic ideas for the regulation of green innovation networks. We employed an undesirable outputs SBM model, social network analysis (SFA), and exploratory spatial data analysis (ESDA) to investigate rules of growth and development, 
structural complexity, and the micro-dynamic mechanisms of green innovation network in Yangtze River Economic Belt from 2010 to 2019. Our conclusions are as follows:

i. The efficiency of green innovation in the Yangtze River Economic Belt has improved significantly but the gap between particular cities has widened. Its spatial evolution showed strong dynamic characteristics from 2010 to 2016, and there has been a problem of efficiency degradation since then. The inefficiency of management and the lack of supervision systems have become a ceiling that hinders the improvement of green innovation efficiency in the Yangtze River Delta region, while the middle and upper reaches of this region have fallen into the trap of the "Matthew effect" due to the lack of endogenous motivation for urban green innovation. During the research period, the spatial heterogeneity of green innovation efficiency was always present, and the heterogeneity and gradualism of local spatial evolution promoted the formation of a unique agglomeration pattern with provincial capitals and central cities as the core.

ii. The density of the urban green innovation network in the Yangtze River Economic Belt has obviously increased and the green innovation network has changed from an initial stage of growth and development dominated by Edge Network characterized by simplicity and sparsity to a rapid growth period dominated by a Basic Network. In the end, this formed a complex network pattern with rich levels and a tight structure. Both structural and hierarchical imbalance in the green innovation network exist at the same time. The preference attachment characteristics of network connections led Chengdu and Chongqing has no connection with the neighboring areas and built connections with more distant areas of the Yangtze River Delta region. This long-distance connection, which breaks down geographical distance and administrative boundaries, helps new network nodes to be incorporated into the overall green innovation network.

iii. The green innovation network in the Yangtze River Economic Belt presents a horizontal " $\mathrm{T}$ "-shaped spatial structure. Guizhou, Yunnan, and western Sichuan, which are located in the marginal areas of the network, have become the key to limiting the complexity and advancement of the green innovation network in the Yangtze River Economic Belt. The total connection strength of the edge layer network is relatively low, however, it accounts for a large proportion of the total number of connections. Eliminating redundant connections and developing Support Network and Core Network has become an effective way to achieve the balanced development of the green innovation network hierarchy. The green innovation network shows reciprocity in its micro self-organizational effects and it has the tendency for a group relationship to evolve with a closed tripartite association.

iv. Divergent nodes under conditions of node symmetry help to form more extroverted connections and to promote the overall network expansion by absorbing isolated nodes or embedding subnetworks. The proximity of nodes leads to an increase in the distance spent on green innovation network connections, which hinders the growth and development of the network as a whole. Connection efficiency can promote the formation of a more stable and resilient network structure and the siphon effect in terms of connection symmetry leads to the improvement in network hierarchy, which results in path locking and structural rigidity. Knowledge flow association within the network drives the flow of new technology, thus promoting the growth and development of the green innovation network.

Further, we extend the results of the research on urban green innovation network in the Yangtze River economic belt to other regions or countries. The evolution of green innovation network is not only affected by transportation, talent flow, trade activities, and technology market factors, but also has a general structural imbalance. Therefore, enterprises with green innovation potential must be selected as key support objects in combination with the economic characteristics and industrial development of the studied region, so as to enhance the supporting role of key core nodes in the complexity of green innovation network structure. In addition, collaborative innovation of green technologies among enterprises in different regions should be encouraged, and the formation of a 
mature cooperation system of inter-regional technological innovation and technology market transactions accelerated. In particular, the edge nodes in the green innovation network should strengthen the connection strength with the core nodes or enhance the ability of independent green innovation, so as to ensure the stability and order of the evolution of the green innovation network in the research area.

Although this study has made progress in the microstructure and self-organization law of green innovation, there is still room for improvement. On the one hand, this study does not deeply discuss the reasons for the evolution of green innovation network structure and regional heterogeneity. Existing studies mainly focus on structural complexity and evolution, lack of exploration of internal mechanism, and there is still a research gap. This paper holds that the structural evolution and development of green innovation network is the result of the game between "groups" composed of network nodes with similar characteristics. Therefore, in future research, we will explore the internal mechanism of the evolution of green innovation network. On the other hand, this study has limitations in the selection of influencing factors of green innovation network. The economic attributes, location attributes, and transportation network of network nodes play a key role in the development of green innovation network. In the follow-up study, we will focus on the impact of other networks and node attributes on green innovation networks.

Author Contributions: Methodology, H.D.; Software and data curation, Z.C. and K.-T.C.; Supervision, H.T. and J.H.; Writing-original draft preparation, R.Z. All authors have read and agreed to the published version of the manuscript.

Funding: This work is partly supported by the by [Shandong Provincial Natural Science Foundation] grant number [ZR2020MG015]. And the APC was funded by [ZR2020MG015]. Any opinions, findings, conclusions, and recommendations expressed in this paper are those of the authors.

Institutional Review Board Statement: This study does not involve human or animal research, so it gives up the ethical review and approval.

Informed Consent Statement: This study does not involve human or animal studies.

Data Availability Statement: This study did not report any data sets.

Acknowledgments: This work was partly supported by the Shandong Provincial Natural Science Foundation for the promotion of the academic excellence of universities under grant No. ZR2020MG015.

Conflicts of Interest: The authors declare no conflict of interest.

\section{References}

1. Guan, D.; He, X. Does the urban sprawl matter in Yangtze River Economic Belt, China? An integrated analysis with urban sprawl index and one scenario analysis model. Cities 2020, 99, 102611. [CrossRef]

2. Zhang, Y.; Sun, M. Decoupling water environment pressures from economic growth in the Yangtze River Economic Belt. China Ecol. Indic. 2021, 122, 107314. [CrossRef]

3. Tang, L.; He, G. How to improve total factor energy efficiency? An empirical analysis of the Yangtze River economic belt of China. Energy 2021, 235, 121375. [CrossRef]

4. Chen, X.; Liu, X. Three-stage super-efficiency DEA models based on the cooperative game and its application on the R\&D green innovation of the Chinese high-tech industry. Comput. Ind. Eng. 2021, 156, 107234. [CrossRef]

5. Qi, X.; Huang, X. The transformation and driving factors of multi-linkage embodied carbon emission in the Yangtze River Economic Belt. Ecol. Indic. 2021, 126, 107622. [CrossRef]

6. Li, K.; Zhou, Y. Decoupling of economic growth from CO2 emissions in Yangtze River Economic Belt cities. Sci. Total Environ. 2021, 775, 145927. [CrossRef]

7. Shu, L.; Wang, T. Summertime ozone pollution in the Yangtze River Delta of eastern China during 2013-2017: Synoptic impacts and source apportionment. Environ. Pollut. 2020, 257, 113631. [CrossRef] [PubMed]

8. Yuan, G.; Ye, Q. Financial innovation, information screening and industries' green innovation-Industry-level evidence from the OECD. Technol. Forecast. Soc. Chang. 2021, 171, 120998. [CrossRef]

9. Peng, H.; Shen, N. Can environmental regulation directly promote green innovation behavior?-Based on situation of industrial agglomeration. J. Clean. Prod. 2021, 314, 128044. [CrossRef] 
10. Zeng, J.; Škare, M. The co-integration identification of green innovation efficiency in Yangtze River Delta region. J. Bus. Res. 2021, 134, 252-262. [CrossRef]

11. Choi, J.O.; Yu, U. Diffusion of innovations in finite networks: Effects of heterogeneity, clustering, and bilingual option on the threshold in the contagion game model. Phys. A Stat. Mech. Its Appl. 2020, 545, 123672. [CrossRef]

12. Luo, Q.; Miao, C. Efficiency evaluation of green technology innovation of China's strategic emerging industries: An empirical analysis based on Malmquist-data envelopment analysis index. J. Clean. Prod. 2019, 238, 117782. [CrossRef]

13. Lyu, Y.; Zhu, Y. Open innovation and innovation "Radicalness"-The moderating effect of network embeddedness. Technol. Soc. 2020, 62, 101292. [CrossRef]

14. Saunila, M.; Ukko, J. Sustainability as a driver of green innovation investment and exploitation. J. Clean. Prod. 2018, 179, 631-641. [CrossRef]

15. Borsatto, J.; Amui, L. Green innovation: Unfolding the relation with environmental regulations and competitiveness. Resour. Conserv. Recycl. 2019, 149, 445-454. [CrossRef]

16. Zeng, W.; Li, L. Industrial collaborative agglomeration, marketization, and green innovation: Evidence from China's provincial panel data. J. Clean. Prod. 2021, 279, 123598. [CrossRef]

17. Wang, H.; Cui, H. Effect of green technology innovation on green total factor productivity in China: Evidence from spatial durbin model analysis. J. Clean. Prod. 2021, 288, 125624. [CrossRef]

18. Abbas, J.; Sağsan, M. Impact of knowledge management practices on green innovation and corporate sustainable development: A structural analysis. J. Clean. Prod. 2019, 229, 611-620. [CrossRef]

19. Liu, C.; Gao, X. Research on regional differences and influencing factors of green technology innovation efficiency of China's high-tech industry. J. Comput. Appl. Math. 2020, 369, 112597. [CrossRef]

20. Wakeford, J.J.; Gebreeyesus, M. Innovation for green industrialisation: An empirical assessment of innovation in Ethiopia's cement, leather and textile sectors. J. Clean. Prod. 2017, 166, 503-511. [CrossRef]

21. Bai, Y.; Wang, J. A framework for determining the impacts of a multiple relationship network on green innovation. Sustain. Prod. Consum. 2021, 27, 471-484. [CrossRef]

22. Zhao, N.; Liu, X. The performance of green innovation: From an efficiency perspective. Socio-Econ. Plan. Sci. 2021, 78, 101062. [CrossRef]

23. Rezende, L.; Bansi, A. Take your time: Examining when green innovation affects financial performance in multinationals. J. Clean Prod. 2019, 233, 993-1003. [CrossRef]

24. Miao, C.L.; Duan, M.M. Spatial heterogeneity and evolution trend of regional green innovation efficiency-an empirical study based on panel data of industrial enterprises in China's provinces. Energy Policy 2021, 156, 112370. [CrossRef]

25. Vidmar, M.; Rosiello, A. New Space and Agile Innovation: Understanding transition to open innovation by examining innovation networks and moments. Acta Astronaut. 2020, 167, 122-134. [CrossRef]

26. Marra, A.; Antonelli, P. Emerging green-tech specializations and clusters-A network analysis on technological innovation at the metropolitan level. Renew. Sustain. Energy Rev. 2017, 67, 1037-1046. [CrossRef]

27. Wang, J.; Xue, Y. Green learning orientation, green knowledge acquisition and ambidextrous green innovation. J. Clean. Prod. 2020, 250, 119475. [CrossRef]

28. Yang, R.; Che, T. The Impacts of production linkages on cross-regional collaborative innovations: The role of inter-regional network capital. Technol. Forecast. Soc. Chang. 2021, 170, 120905. [CrossRef]

29. Gonzalez, E.B.; Easdale, M.H. Socio-technical networks modulate on-farm technological innovations in wool production of North Patagonia, Argentina. J. Rural Stud. 2021, 83, 30-36. [CrossRef]

30. Owen, R.; Brennan, G. Enabling investment for the transition to a low carbon economy: Government policy to finance early stage green innovation. Curr. Opin. Environ. Sustain. 2018, 31, 137-145. [CrossRef]

31. Peng, W.; Yin, Y. Spatial spillover effect of green innovation on economic development quality in China: Evidence from a panel data of 270 prefecture-level and above cities. Sustain. Cities Soc. 2021, 69, 102863. [CrossRef]

32. Liu, Y.; Shao, X. Spatio-temporal evolution of green innovation network and its multidimensional proximity analysis: Empirical evidence from China. J. Clean. Prod. 2021, 283, 124649. [CrossRef]

33. Chen, F.; Liu, H. How does integration affect industrial innovation through networks in technology-sourcing overseas M\&A? A comparison between China and the US. J. Bus. Res. 2021, 122, 281-292. [CrossRef]

34. Desmarchelier, B.; Djellal, F. Which innovation regime for public service innovation networks for social innovation (PSINSIs)? Lessons from a European cases database. Res. Policy 2021, 50, 104341. [CrossRef]

35. Teng, T.; Cao, X. The dynamics of inter-firm innovation networks: The case of the photovoltaic industry in China. Energy Strategy Rev. 2021, 33, 100593. [CrossRef]

36. Fan, J.; Xiao, Z. Analysis of spatial correlation network of China's green innovation. J. Clean. Prod. 2021, 299, 126815. [CrossRef]

37. Zhou, M.; Govindan, K. How fairness perceptions, embeddedness, and knowledge sharing drive green innovation in sustainable supply chains: An equity theory and network perspective to achieve sustainable development goals. J. Clean. Prod. 2020, 260, 120950. [CrossRef]

38. Sun, J.; Zou, H. Game analysis of dual network embedded enterprise technology innovation: Intelligent manufacturing pilot perspective. Microprocess. Microsyst. 2020, 103399. [CrossRef] 
39. Yao, L.; Li, J. Urban innovation and intercity patent collaboration: A network analysis of China's national innovation system. Technol. Forecast. Soc. Chang. 2020, 160, 120185. [CrossRef]

40. Yang, Z.; Chen, H. How does alliance-based government-university-industry foster cleantech innovation in a green innovation ecosystem? J. Clean. Prod. 2021, 283, 124559. [CrossRef]

41. Zhang, R.L. Evaluating ecological efficiency of Chinese industrial enterprise. Renew. Energy 2021, 178, 679-691. [CrossRef]

42. Yang, Y.; Guo, H. Flood vulnerability and resilience assessment in China based on super-efficiency DEA and SBM-DEA methods. J. Hydrol. 2021, 600, 126470. [CrossRef]

43. Zhou, W.; Su, D. When do strategic orientations matter to innovation performance of green-tech ventures? The moderating effects of network positions. J. Clean. Prod. 2021, 279, 123743. [CrossRef] 